\title{
The Voice in the Machine: Oral History and Making the Computer Relevant
}

\author{
Thomas Lean \\ British Library \\ Thomas.Lean@bl.uk
}

\begin{abstract}
From the beginning computer history has often been more about technical developments than it has been about the social history of the computer and its effects. This paper describes how a greater attention to the social context of developments, representations of technology, the importance of users, software, and other topics, has presented a number of other ways to make computer history relevant rather than concentrating on the machine itself. This paper considers computer history through the medium of oral history, using interviews collected by National Life Stories at the British Library as part of An Oral History of British Science.
\end{abstract}

Keywords: computer history, social history, oral history, life stories, interviews.

\section{Introduction}

Given the great complexity of the typical computer it is perhaps of little surprise that the machine itself, in all its technical glory, has cast a long shadow over the history of computing. From its early days computer history has often been more about technical developments far more than it has been about the social history of the computer and its effects. In more recent years, the body of literature concerning the history of the computer from other perspectives has grown, taking in, for example the role and culture of computer users ${ }^{1}$, the computer as a business or information machine ${ }^{2}$, the role of the military in the development of computing ${ }^{3}$, and so on, moving the field on from what has sometimes seemed to be a hagiography of dead white machines. This greater attention to the social context of developments, representations of technology, the importance of users, software, and other topics, has presented a number of other ways to make computer history relevant rather than concentrating on the machine itself.

In this essay I consider computer history through the medium of oral history, using interviews collected by National Life Stories at the British Library as part of An Oral

1 See, for example, Christina Lindsay, "From the Shadows: Users as Designers, Producers, Marketers, Distributors, and Technical Support." In How Users Matter: The Co-Construction of Users and Technologies, edited by Nelly Oudshoorn and Trevor J. Pinch. MIT Press, 2003.

2 See, for example, Martin Campbell-Kelly and William Aspray, Computer: A History of the Information Machine, Second edition ed. Westview, 2004.

3 See, for example, Paul N. Edwards, The Closed World: Computers and the Politics of Discourse in Cold War America, MIT Press, 1996. 
History of British Science ${ }^{4}$. Launched in 2009, this ongoing project has collected nearly one hundred life story interviews with British scientists and engineers, drawn from a great variety of fields. As history of technology has long preached, technologies have different meanings to different groups, and the computer is no exception to this. Amongst the interviews in the collection are recordings of computer designers, theoretical computer scientists, programmers, and many scientists and engineers who made use of computers in various different fields, presenting many different perspectives on the machine.

Interviews with figures from the history of computing are nothing new. To give a few examples, in the 1970s the NPL computer scientist and psychologist Christopher Evans carried out a series of interviews with computer pioneers about their work. Several organisations, notably The Computer History Museum, maintain extensive collections of oral history, and short interviews also frequently feature in IEEE Annals of the History of Computing often focused on particular issues that interviewees were involved with. On An Oral History of British Science, the development of computing and computers is not the focus of our interviews, which are essentially biographical in nature. This gives our interviews a slightly different perspective to ones more narrowly focused on particular topics, more concerned with an individual's day to day activities, and broadens the questions to include a wide variety of contextual details. Like any source, oral history can have its inaccuracies and biases, it needs to be subject to the same scrutiny as we would any other document and used in concert with other sources for serious academic use. These reservations aside, it is an excellent way of conveying the feel of a place or time, uncovering human interactions around technology that are undocumented elsewhere, and putting a voice to technical developments. In short it presents a number of ways to help make the history of computer relevant to wider audiences.

My aims in this paper are threefold. I touch on computer development as recalled from the point of view of designers, to explore how telling the stories of computers helps us to appreciate them as works of human endeavour rather than as monolithic artefacts. Secondly, I draw on interviews with computer users to suggest how oral history can add a sense of how people interacted with computers in the past, and demonstrate their relevance by uncovering ways in which the computer has shaped the world around us through its impact in science and engineering. Thirdly, and throughout, I aim to demonstrate some of oral history's uses in conveying the story of computing, not just as a historical source to be analysed and interrogated, but as a way of telling computer history in a way that forefronts the people involved and adds context to the machines themselves.

\section{Machine Builders}

We generally encounter old computers as complete artefacts, things that have been given shape by human activity. However, the inescapable presence of these complete

\footnotetext{
4 An Oral History of British Science, http: / /www.bl.uk/aboutus/stratpolprog/ oralhist/oralhist.html Most of the interviews referred to in this article are available through British Library Sounds, sounds.bl.uk/ [accessed 6 August 2013]
} 
artefacts sometimes obscures our view of the processes that created them. The approach of the life story interview encourages not just descriptions of old technology but narratives about development and use. Oral history offers a way of deconstructing finished computer objects by asking designers to tell us the story of how they were built. For example, our interviews with hardware designers reveal the step by step process of building early computers; starting with an outline plan, designing units in detail, having them constructed, adding them to the whole prototype. The computer, our interviewees explain at length, is the result of a gradual process of a machine taking shape, as Geoff Tootill, one of the developers of the 1948 Manchester Baby computer recalled of its construction:

The very last thing we could contemplate doing was to design the whole thing and have it all built and wire it all up and then find out why it didn't work. It was essential that the whole thing would work together at every stage as you added one unit after the other. [...] It was necessary that the apparatus should do something which we could see was correct, or if it wasn't correct we could mend it until it was correct at every stage, every chassis that we connected up.

[...]

Well, we went on with this process of adding the units and making the whole lot do something together at every stage until we got to the stage when we'd made a computer. ${ }^{5}$

Along the way, in the extended versions of these bit-by-bit stories we learn many other contextual details about their construction: technical challenges that had to be overcome; details of interactions with colleagues; the role of lesser known people, such as technicians; amusing anecdotes; the principles that the machines were built on; their important design details; and the human activity involved in development. They give us the material to consider complete vintage computers as the culmination of processes and various human activities rather than as monoliths. For example, computer designer Raymond Bird recalled how the logical design of the ICT1301 was checked by rather unconventional means:

So this machine had masses of gates and shifting registers and all that sort of thing in it, and in order to check over the logical design John Wensley very sensibly said, 'We're going to mechanise, humanise, this, we're going to draw out the whole of the logic design on the conference room floor,' [...] And then human beings acted as pulses. A pulse of information, a voltage which lasts for say a millisecond. So a computer consists of pulses tearing all around the place at a megacycle. So people were stood representing pulses at the correct place in the circuit and then in order to check that a particular operation is correct, say an addition, the number being added would be a stream of pulses, one after the other. [...] There'd be a row of these human beings and they would march onto the machine [...] and told to move to the next point round the machine [...], with each time seeing that the effect of them being at that point at that time

\footnotetext{
5 National Life Stories interview with Geoff Tootill. C1379/02
} 
didn't clash with another one, or they'd got somewhere to go and it wasn't shut off to them. ${ }^{6}$

Not only does this insight reveal something of the human story behind the ICT1301, but it also helps to explain some the technicalities inherent in the machine. We learn how information travels about within it as electrical pulses, in an engaging non-technical manner. Whilst the interviews are often rich sources of technical detail about historic developments, our approach has been to try and encourage interviewees to discuss their work in ways that make it intelligible to the general public as well as experts wherever possible. Given that the complexity of computing is something of a barrier in interesting people in its history, this approach presents some promise as a way of explaining technicalities in an engaging manner. While not always as polished as historians' considered explanations, there is a unique value in having technology explained by people who helped create it, particularly when it integrates with an engaging historical story or anecdote.

Of course discussions of technicalities are not the only insight we get from computer designers, oral history allows us to ask for reflections about the work they were involved with, revealing thoughtful insights into how the future of computing looked from the past and adding a further human dimension to stories of machines. The much hackneyed anecdote about IBM's Thomas Watson declaring that the world only needed five computers, is so attractive not only because its amusing when important people get things wrong, but also because it causes us to reflect for a moment on the comparison between then and now. Discussing the motivations and expectations of computer designers for their machines has something of the same appeal because the comparison can be so engaging. Geoff Tootill, for instance recalled his own modest expectations for computing back in 1948 when he was helping to develop the first stored program computer in Manchester.

We thought the culmination of our work would be a large scale computer with not only facilities for subtraction, but also addition, multiplication and division, and a much bigger storage capacity and also a capacity for storing big programs... So we thought that such a large computer would be required for several different tasks, like weather forecasting for example, because we knew that a competent meteorologist could compute what tomorrow's weather was going to be based on today's available data, but unfortunately it would take him a week or a fortnight at the least. So the idea was that a big computer could do this computation in time to give a useful answer about tomorrow's weather. And there were no doubt other tasks like atomic energy calculations. So that we thought there would be scope for another, one or perhaps two big computers in the UK and three or four in Europe and probably half a dozen in the US because they always have big ideas in the US. ${ }^{7}$

Reflections such as these cause us to pause and think about the nature of computing in the past, and how it compares from our reference point of computing in the present day.

\footnotetext{
${ }^{6}$ National Life Stories interview with Raymond Bird. C1379/04

7 National Life Stories interview with Geoff Tootill. C1379/02
} 


\section{Users}

As Christina Lindsay points out in a useful essay on the co-construction of the TRS80 personal computer, designers' conceptions of what the user will do with their computer, can differ sharply from what users actually end up doing. ${ }^{8}$ The computer is the universal machine, open to a wide range of interpretations and applications; they are not ends in their own right. They are tools to be used for other purposes, a means to an ends, as Professor Sir Maurice Wilkes reminds us in his interview:

When I started building the EDSAC I had no doubt about who were going to be our users, they were people like myself. I mean I used to be doing things that would take perhaps a week. Solving a set of ten by ten equations took about a week with a desk calculator machine. Well that could be done rapidly with a digital computer. ${ }^{9}$

Computer use can be a particularly difficult subject to convey. Whilst the development of computer hardware has at least left obvious, readily interpretable, artefacts behind, the history of computer use and software is rather more ethereal. Punched cards, magnetic tape, and program print outs are perhaps not the most engaging of artefacts, and how much they can adequately represent the history of software and use is debatable. Furthermore the arcane dead programming languages and the complex principles they work on are not easy to convey to wider audiences. Oral history's ability to convey human feelings and activities once more gives us a powerful way to understand historical computer use on a number of levels. For example Stephanie Shirley, an early computer programmer and later pioneer of home working in the software industry, impresses much on the reader the fascination of programming early computers:

Well it has some of the beauty of mathematics, it has a lot of logic in it. It has a sort of puzzle element to it. In fact the sort of early, what do they call it, aptitude tests that they used for programming were things like, as they had used for Bletchley Park coding, things like, people who played with crossword puzzles, people who play chess. It's those sorts of, slightly quirky puzzle-solving capabilities. And, programs would take, well, obviously there were different sizes and so on, but, sometimes you would be working on, on a program for months and months and months, so the satisfaction when you did actually complete it and check it, and, most of that time was spent in checking, where you sort of, are nearly there, just got one more bug to get out, made for a very fulfilling life really, that you realised that you had actually created something. ${ }^{10}$

Computing, as this neatly demonstrates, is about people's interactions with the computer. Recollections of using old computers have the ability to bring dead machines back to some sort of life by capturing the day to day activity that happened around them. Our interviews convey use - the methodical process of checking code, the frustrations of poor reliability, the parsimonious care taken to write programs to fit

${ }^{8}$ Christina Lindsay, "From the Shadows: Users as Designers, Producers, Marketers, Distributors and Technical Support."

9 National Life Stories interview with Professor Sir Maurice Wilkes. C1379/21

${ }^{10}$ National Life Stories interview with Dame Stephanie Shirley. C1379/28 
into small computer memories not as simple facts, but as individually recalled human experiences. The following impression of 1950s computer programming from Ferranti programmer Mary Lee Berners-Lee wonderfully demonstrates how the history of computer use can be enlivened by personal reflection and details.

It's very difficult getting a computer program right, even when one used to do what they called a dry run, imagine work right through your program, going through it instruction by instruction to see what actually happens to the numbers and you pick up a lot of errors like that, and then you go along and try it on the computer and it doesn't work and you're very surprised and you think it's the machines fault and sometimes it was because the machine was not reliable, anything but. But it's more likely in fact to be your fault [...] The program was typed up on punched teleprinter tape, five hole tape, and if you had to make an alteration we'd cut out tiny little bits of sellotape, coloured sellotape, and stuck them over the hole and had little hole punches with which you could punch a new hole if you wanted one and turn pieces of sellotape to cover up the holes you didn't want. And they tended to come off so you thought you'd got rid of a fault and there it came again, and you found your little bit of sellotape had come off, so you were wise to stick sellotape on the both sides. ${ }^{11}$

Use, as this example shows us may not be about complex programming concepts, but about easier to relate to human activities, which may make a good starting point for more detailed discussion and explanation of the more complex principles involved.

Even when machines have been restored to use, we generally encounter old computers today in rather sanitised settings. There are of course exceptions, but the museum gallery is rarely like the machine room or computer development lab of decades ago. Vintage photos and films give us some feel for these machines in their original surroundings, but oral history provides other insights and reflections. From our interviews, computer history gains a sense of place, amidst diverse settings ranging from the decrepit barn where Andrew Donald Booth built some of his earliest computers in the 1950s, to the palatial splendour of Ferranti's Portland Place computer centre, and many other places besides. Not only do we gain descriptions of environments but insights into the personal interactions and social history that was happening in them, for example rocket scientist Bob Parkinson's recollections of using the Rocket Propulsion Establishment's computer in the 1960s and 70s.

The other way that knowledge transferred round the establishment at that time, not just scientific knowledge but more the knowledge of what the hell was happening, was the computer. In those days it was an Elliott 803, filled a large room with air conditioning, and you ran your programmes by taking a piece of punch tape down there and getting them fed into the machine and getting a piece of punch tape come out which you then went to a teletype machine and printed out. So I used to think of it as down by the river... doing the washing. So there was - there was a queue which we stood and chatted to one another and so anything that was happening got round and that went between division, because it was the computer for the whole thing and you weren't sat at your own little VDU, you were actually meeting other people who were

\footnotetext{
${ }^{11}$ National Life Stories interview with Mary Lee Berners-Lee.
} 
also running computer programs for different purposes. So yes, that certainly at the time acted as a way of, an informal way, of finding out what was going on ${ }^{12}$.

\section{$4 \quad$ Wider Effects}

In such ways oral history has the ability to help put vintage computers back into their original contexts of use and help us to understand the human interactions that went on in these spaces. However, use goes beyond these immediate contexts to have wider effects. We are often told that one of the key effects of computers has been to speed up calculations, however speeding up the solving of sums is often only a means to an ends. What difference that speeding up of calculations makes is often far more dramatic and far more relevant to understanding computing in a broader sense. As Jon Agar has explored, early computing had an important role to play in several other branches of science, but this was not always just a straight forward matter of speeding up science ${ }^{13}$. Our interviews have explored the differences computing made in several branches of science and engineering, including civil engineering, aeronautics, materials science, geophysics and others, to cover a range of different perspectives on the influence computing made in different fields.

What emerge from these accounts are not always straightforward Whiggish stories of the computer improving things. Rather, we gain more complex accounts with issues to think over, that help to nuance our understanding of the differences computing has made and of the interaction of computing with work in other fields. For example, engineers often talk about having a feel for their work, an instinctive understanding of how mechanisms function or how stresses are distributed in structures. Engineering is not a theoretical activity, but one about the application of knowledge in practical ways, testing that knowledge with experiments and calculations along the way, and building up a sort of tacit understanding of whatever structure is being built and a confidence it will work.

Before computers, doing such work was not necessarily easy or quick, but it provided the all-important 'feel' factor. Structural engineer Michael Parsons, who made key contributions to the design of the Severn Bridge, recalled that back in the 1950s to analyse the effects of a side wind on a suspension bridge involved solving considerable numbers of simultaneous equations with a slide rule.

To do five simultaneous equations it used to take me about two hours. Of course now we do it in milliseconds. But I think the position now is that when I was doing this it took me two hours to get an answer and I jolly well had a feel for what the structure was doing. I understood it and I felt the structure was almost a part of me.

As Parsons continued, for later designers having the computer do the sums may have speeded things up dramatically, but for engineers used to painstakingly building up tacit understanding of their structures, it risked taking something away.

Whereas later on people would use a computer programme for analysing suspension bridges and they'd put all these loads on and they'd press the

\footnotetext{
${ }^{12}$ National Life Stories interview with Bob Parkinson. C1379/68

${ }^{13}$ Jon Agar, 'What difference did computers make to science?', Social Studies of Science (2006).
} 
button and they'd get all the answers out, pages and pages of answers. But I always had the feeling that they didn't really know what they were looking at. Whereas I knew that, if I changed something, I knew what sort of effect it would have. ${ }^{14}$

As other interviewees have related, taking designers a step away from fundamentals and black boxing the calculations in a program, sometimes risked mistakes, that went unnoticed. Bridge designer Peter Head, for example recalled that as computer use moved on from engineers devising their own programmes to solve their problems to using models to predict the performance of bridges potential risk emerged:

People started to use them as black boxes. And I can still remember to this day a particular example, where a young engineer had analysed a bridge and gone all through the detailed design of a bridge and had come up with solutions, which weren't quite right. They didn't look quite right. And so I questioned it and went back and found that there was a fundamental error in the original coding of some of the members of the bridge, which had never ever been questioned. [...] And it always worried me that people tended to use computer models as black boxes and I worried that engineers would lose the ability to think at a fundamental level about structural behaviour. ${ }^{15}$

As these examples show, oral history is an excellent way of conveying some of the issues raised by the use of computers in wider science and technology. It also gives us an idea of how computing fitted into scientific and technical organisations and how the people within them saw the coming of computers. For example as Ralph Hooper, who led the early design of the Harrier jump jet, recalled the first computer at the Hawker Siddeley site he worked at:

We got our first computer in 1958, it was a Ferranti Pegasus computer by chance. And it was nearly the size of this room, not quite as long perhaps. And it was done by batch processing I think they called it, and they got a stack of work together and then fed it through the computer, only the mathematicians were allowed to use the computer and at that time the head of maths - there was a small maths section, I forget how many there were in there, but he was dead scared of this damn computer and he got into a great tizzy every time he had to go in and use it. His number two was a much calmer chap and he made best use of it. ${ }^{16}$

As this example suggests, computers as recalled by interviewees from outside computing do not always seem to be a dramatic and important part of scientific workplaces. Indeed, the computer sometimes comes across as an evolutionary step rather than a revolutionary development, just a better tool to do things they were already doing anyway, for instance the replacement of slide rules, by scientific calculators, by personal computers, for individual engineers' on the job calculations. In many of our interviewees with those from different fields, the computer seems rather everyday, not unimportant, but part of the fabric of the workplace and part of

\footnotetext{
${ }^{14}$ National Life Stories interview with Michael Parsons. C1379/77

${ }^{15}$ National Life Stories interview with Michael Parsons. C1379/79

${ }^{16}$ National Life Stories interview with Ralph Hooper. C1379/27
} 
the stories of other technologies. It is precisely this part in the stories of other technologies that help these accounts to demonstrate the wider relevance of computing, by tracing its influences through to items in the world around us.

Jet engine designer Ralph Denning, for instance, recalled how collected data about previous designs allowed Rolls-Royce Bristol to work out the fundamentals for designing a jet engine and create software that could quickly produce outline designs for new jet engines. Detailed designers could work these up to full designs, but they also provided a way of quickly developing an optimum design, coping with a large volumes of work, and producing a range of weighed and costed designs for customers. Another civil engineer, Ron Bridle, recalled the flexibility offered by iteration using the computer:

And iteration, the one thing the computer can do that other systems can't do is iterate and iterate and iterate and iterate. And that's a powerful, powerful method then. So you can use methods you couldn't possibly use longhand.

As Bridle continued, this iteration was useful in the development of standard, optimal, engineering components and in planning road layouts:

You could move the [road] alignment, like this, keep iterating, moving the alignment until you could get minimisation of travel costs, and going up and down, minimisation of excavation costs. ${ }^{17}$.

In such ways, oral history with computer users from other fields opens up new ways of appreciating the relevance of computing by showing attitudes towards it more widely, and by giving us examples of its use that link the computer to other points of reference. This linking of the computer's abilities to other things in the wider world can suggest other ways of demonstrating complex principles. For example, iteration goes from being a function of the computer to being about how one decides where to build a road. In such ways we move away from an understanding of the computer as a large complex machine, to one that emphasises its influence in familiar everyday things and in shaping the world around us.

\section{Conclusions}

As the recent attention to the Alan Turing centenary has amply demonstrated, personal stories can be useful way of interesting a wider public in the history of computing. In this paper I have explored ways in which personal stories gathered from oral histories can help to make the history of the computer more engaging than just focusing on technology. In particular I have suggested how the retelling of stories about early computing development can help us to de-construct computer artefacts, helping us to appreciate the human stories that lie hidden behind them. I have given examples of how oral history can open up a world of past human interactions with the computer. I have suggested how technicalities can be engaged with by anecdotes or personal reflections, and how attention to computer use can illustrate the social history of computing, and demonstrate the relevance of computing more widely than as just 'computer history'.

${ }^{17}$ National Life Stories interview with Ron Bridle. C1379/75 
Through all of this I have presented ways for people to appreciate the computer as something other than a complex item of technology. Descriptions of the computer as it was when it was in use, the emotions and expectations of designers, and the difference it made to users, all have the potential to act as 'hooks' with which to catch the attention of audiences for whom understanding complicated machines is passé. Oral history reminds us that the history of computing is not just about computers, but about the interaction of computers and people. Hearing stories of computer history, from the people who helped to make that history happen in their own words, is a uniquely personal way of telling the history of computing. It provides us with a powerful way of interpreting computer history for wider audiences and a way of bringing dead computer machinery to life. 